\title{
ANDES: Evaluating mapping strategies with synthetic programs
}

\author{
João Paulo Kitajima ${ }^{a, *}$, Brigitte Plateau ${ }^{b}$, Pascal Bouvry ${ }^{c}$, Denis Trystram ${ }^{\text {n }}$

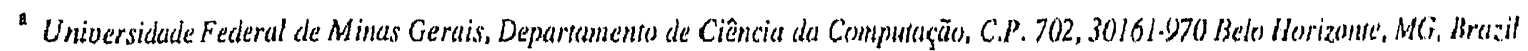

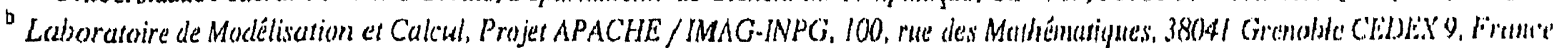

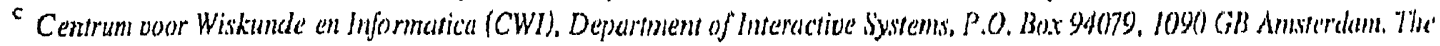 \\ Netherlinds
}

Received I June 1995; revised 19 March 1996; acecpted 26 April 1996

\begin{abstract}
This paper presents the ANDES performance evaluation tool. ANDES is bnsed on the synthetic execulion of parillel programs and it is used for the evaluation of mapping strategies. The Meganode, a distributed memory parillel computer, is considered as our target architecture. ANDES takes into account a benchmark of quintitative models of puralle: ulporithmis and a set of mapping strategies (greedy and iterative algorithnis are use(l). We show how this tool allows un extensive comparison of mapping strategies by using the benchmark, the mapping strategics and different cost functions.
\end{abstract}

Keywords: Performance evaluation; Parallelism; Tool; Synthetic execution; Mapping; Multicomputer

\section{Introduction}

Distributed memory parallel machines (DMPM) are the current trend of high-performance parallel computers. They represent a good balance between cost and performance, mainly because of the connection of several commercial, general and relatively cheap microprocessors. A distributed memory parallel machine is a computer composed of autonomous processors connected by a high speed communica-

\footnotetext{
"Corresponding nuthor. Email: kitajima@dcc.ufing.br.
}

tion network. Each processor has its own adkress space. The Intel Paragon, the Thinking Machines CM-5, the IBM SP and the (ruy TBI) are lypical examples of this generation of parallel computers. The different processing elements inside such a par. allel machine simultaneously execute different pieces of the parallel code, and each piece demands some machine resources, like the processor itself, the memory and the inter-processor communicaliun min" dia. The low level programming paradigm imposited by this kind of architecture is based on messige passing, considering that no momory is physically shared by the processors 
Although more abstract programming models have been developed for DMPMs (e.g., paradigms based on data parallelism, remote procedure calls, logical and functional programming, etc.), the programmer, the compiler or the operating system is always faced with the problem of choosing which processor should execute each of the different pieces of the compiled parallel program. In this allocation problem, known as mapping, the workload is represented by a quantitative and structural model of the parallel program to be executed. On the other side, the DMPM is composed of a set of specific resources: processors with a certain processing power (e.g., expressed in MFLOPS or MIPS), distinct memories with a finite capacity and a communication network with a defined bandwidth. Finally, one or more performance criteria (like execution time or processor load) should be optimized.

The inputs of a typical mapping are a model of the parallel program and one of the parallel computer. These entities, mainly the DMPMs, can however be very complex and their respective models can be somewhat far from the reality. Therefore, it would be interesting to compare the value of a performance criterion computed by the mapping algorithm against the same criterion measured from a true execution of the parallel program on the real DMPM. It is desired, however, to stay in the domain of performance prediction, that is, evaluation should be done without executing the real parallel program on the real parallel machine. It is well known that coding and debugging of parallel algorithms are a quite expensive task.

ANDES is a tool that supports performance evaluation of parallel programs at the prediction level, which considers the existing complex overheads of parallel computers. This is achieved through the use of synthetic parallel executions directly on the parallel machine. In a synthetic parallel execution, the resources of the DMPM are used in a controlled way, but no code is generated. All the steps from the interpretation of the parallel program and from the interpretation of the parallel machine model to the synthetic execution on the target parallel machine are automatically managed by ANDES. ANDES finally computes performance indices from the execution of that workload implemented according to mapping and/or scheduling strategies. Synthetic execution is the chosen performance technique due to the easy control of parameters as well due to the used real environment. The idea is to conjugate the best of the model approach with the best of the real environments.

The next section presents related work. Next, the mapping problem and some strategies used to solve it are introduced. Two aspects of ANDES are then explained: the parallel algorithm modeling language and the synthetic execution manager. A comparison of the mapping algorithms is presented in order to show that the tool is useful. Finally, some conclusions and perspectives are presented. ANDES is an evolution of the ALPES environment presented in [8], which was based on the generation of source files of synthetic programs. The new approach is based on a more efficient synthetic execution, controlled by a kernel that accepts a synthetic workload described in an intermediate format.

\section{Related work}

Any parallel programming environment (e.g., PPSE [10]) can be used to generate synthetic programs. The drawback in using these general environments is that the generation is not automatic. On the other hand, some computer-aided tools use specifically the synthetic approach and, then, a synthetic workload is automatically generated. Three environments can be considered representative: MIMD, $O L G A$ and HASTE.

\subsection{MIMD}

MIMD (Multiple Instruction stream, Multiple Data stream) [3] is a simulation system developed on top 
of DEMOS, a discrete event modeling package written in Simula. MIMD runs on Sun workstations and it simulates the execution of message-passing parallel programs on arbitrary distributed memory multiprocessors. The parallel program (assumed to be correct) is modeled as a directed graph where vertices represent processes and the arcs represent unidirectional channels. A process can execute only four types of statements: compute $(n)$ (compute spending $n$ CPU cycles), sleep $(n)$ (sleep for $n$ seconds), $\operatorname{send}\left(C_{i}, n\right)$ (send message of length $n$ bytes through channel $C_{i}$ ) and receive $\left(C_{i}\right)$ (receive a message on channel $C_{i}$ ). The parallel machine is represented by an undirected graph where the vertices model the processors and the edges model the hard links. All the processors are of the same type (homogeneous). Additional information concerning the characteristics of the processors and of the links are given. Process-to-processor mapping is done through specific procedures. The simulation produces performance information like processor/link usage and process/channel activity. Global snapshots can be obtained without interfering with the execution simulation. MIMD is used inside a larger experimental context. In this context, there is a program generator that automatically generates the synthetic program from an experiment control file, a process model (defining a particular programming paradigm), and a software graph generation strategy (random graphs are used in [3]).

\subsection{OLGA}

OLGA (Occam Load Generation Application) [18] is an environment for performance evaluation based on synthetic programs derived from a skeleton file (the synthetic task: describes the sequence of computation and communication phases) and from a parameter file (describing the kind and duration of computation phases and sizes of the data exchanged between processes). A skeleton file is an Occam process containing the code executed on each pro- cessor. This skeleton is obtained from a skeleton library associated with BACS, the Basel Algorithm Classification Scheme. BACS provides a base for the description and classification of parallel algorithms. OLGA is composed of four parts: (1) the parser scans the parameter file in order to determine the workload; (2) the library contains the algorithm skeletons, process interaction structures, basic data structures, random generators, load process primitives and other global services; (3) the loader loads the code and the (probabilistic and dynamic) distribution tables, and (4) the frame executes on each processor. It is responsible for time measurements, for the execution of the computation and communication loads. OLGA currently executes on a Transputer network and it is used to evaluate ParStone, a synthetic benchmark based on BACS [18].

\subsection{HAMLET}

HAMLET is a computer-aided environment developed to support the design of parallel industrial applications. Emphasis is given to the early stages of the design, considering that "the later a mistake is detected, the more expensive it is to fix" [15]. Currently, HAMLET is composed of three modules: (1) the Design Entry System (DES) that allows the specification of the application, the target hardware and the mapping of the software on the hardware; (2) HASTE (HAmlet Simulator Tool (E)), a tool that simulates (by discrete events) the execution of the application (described with the DES) on a (also simulated) parallel machine, and (3) TATOO, a graphical interface used to analyze the tracefile generated by the simulation. The Design Entry System allows the specification of "delays" that model synthetic processor workload. HASTE is a more general tool than $M I M D$, since the first tries to embed the synthetic approach in a wider context of parallel software engineering. MIMD and OLGA (and, as we will see next, $A N D E S$ ) are more performance evaluation oriented, although they eventually can be used 
in a more general tool for supporting the development of parallel programs.

As presented above, other environments using the synthetic approach are available, but only OLGA is comparable with ANDES, considering that a synthetic execution on a real parallel machine is done. MIMD and HASTE use simulators, not a real paralle] system. The simulation is only interesting if the parallel computer is accurately modeled, mainly the contention generated when using the machine resources.

\section{The mapping problem}

the main goal of a good mapping is to minimize the execution time of the whole program. Other objectives can also be achieved, for instance in a multi-user processor network, it is interesting to minimize the average use of the different resources. The goal to be reached is often represented by a cost function.

Let us denote:

$T$, the set of tasks,

$P$, the set of processors, calc $(t)$, the total computing time of task $t$, $\operatorname{comm}\left(t, t^{J}\right)$, the total communication time between $t$ and $t^{\prime}$,

the function alloc $(t)$ returns the processor where task $t$ is allocated.

Formally defined, a mapping is an application from $T$ to $P$ which associates to each task a unique processor. The cardinality of all possible solutions is $\mid T^{|P|}$. Even if this number can be slightly decreased due to some symmetry considerations, it remains too large for practical problems.

Several algorithms can be found in the literature for solving the mapping problem. We can roughly distinguish two classes of methods, namely, exact algorithms and heuristics [4]. Exact algorithms can only be used when the space of solutions is small enough, for instance when only a few tasks have to be allocated to a machine with a small number of processors. Exact algorithms give the optimal solutions but in practical cases they cannot be used because of the combinatorial explosion of the number of solutions.

The goal of heuristic algorithms is to give good solutions in relatively reasonable time. Two subclasses of heuristic algorithms have mainly been explored: greedy algorithms which progressively construct the solution, and iterative algorithms whose principle is to improve an existing solution. Random mapping is also an alternative and generally supported by known environments (eg., PVM [17]). However, better mappings can be found if some knowledge is acquired from the application structure and behavior. Naturally, the time to treat this knowledge should not be greater than the time gained with this supposed better mapping.

\subsection{Greedy algorithms}

In a greedy algorithm, the mapping is done without backtracking (a choice already made can never be reconsidered). The allocation of the $i$-th task is based on a criterion depending on the mapping of the first $(i-1)$ tasks. Two kinds of greedy algorithms can be envisaged for the mapping problem: the first ones are based on empirical methods and the second ones come from the relaxation of classical graph theory algorithms which are optimal for some restricted cases.

Examples of such criteria are given below:

- LPTF (Largest Processing Time First) is a heuristic whose criterion is restricted to load balancing. It is well-known that its performance in the worst case is about $4 / 3$ from the optimal when considering independent tasks [9].

- Lo presents in [12] an algorithm based on a maximal matching which minimizes the costs of 
communications between tasks. This algorithm is optimal for UET (Unitary Execution Time) tasks if the number of tasks is less than twice the number of processors and if at most two tasks are allocated to one processor.

- Algorithms based on a minimal cut of bi-parted graphs can also be used $[12,16]$.

Greedy algorithms are easy to implement and have a polynomial complexity (often less than $O\left(|T|^{3}\right)$, for instance LPTF is of $|T| \log |T|$ complexity).

\subsection{Iterative algorithms}

All iterative algorithms start from an initial solution and try to improve it. Note that the initial solution can be found using a greedy algorithm. Usual iterative algorithms try to exchange tasks between processors to locally improve the solution. Most such algorithms use random perturbations to leave local minima and to jump to better solutions.

A well-known iterative algorithm is the Bokhari algorithm [1]. Its cost function (called cardinality) takes into account the number of tasks correctly mapped on the processor network and uses pair-wise exchanges of tasks to improve it. The basic hypothesis is that the number of tasks must be equal to the number of processors. Grouping methods must be used to take into account a greater number of tasks.

\subsubsection{Hill climbing}

The basic iterative algorithm, called hill climbing consists of starting from a given solution and to improve it iteratively using a neighborhood relation. This solution leads directly to a local optimum.

\subsubsection{Simulated annealing}

One of the most popular iterative methods is simulated annealing $[2,14]$. This method is based on an analogy with statistical physics: The annealing technique allows a metal with the most regular struc- ture as possible to be obtained. It consists of heating the metal and reducing the heat slowly so that it keeps its equilibrium. When the temperature is low enough, the metal is in a equilibrium state corresponding to the minimal energy. At high temperature, there is a lot of thermic agitation which can locally increase the energy of the system. This phenomenon occurs with a given probability decreasing with the temperature. It corresponds mathematically to giving a chance to leave a local minimum of the function to optimize.

\subsubsection{Tabu search}

Tabu search is an iterative meta-heuristic [6]. It tries to find the best neighbor of a given solution. To avoid cycling and local optima, a tabu list is established. This tabu list contains information concerning the last moves. A tabu move is not allowed except if an aspiration criterion is satisfied (i.e., if the proposed neighbor gives a better value of the objective function).

\subsection{Mixed strategies}

The two preceding classes of heuristic mapping algorithms are not the only ones but are the most present in the literature. Other solutions can be obtained by mixing the preceding algorithms: an initial solution could be obtained by simulated annealing and then tabu or algorithms like the branch \& bound algorithms [7] could be used to improve the mapping.

\subsection{Quality of the solution}

Most solutions of the mapping problem are based on the optimization of cost functions. Let us denote by $z$ such a function.

Under the previous assumptions in the model, we have two opposite criteria to take into account: (1) minimizing inter-processor communications, and (2) balancing the computations between processors. 
These criteria are opposite in the sense that minimizing external communications leads to group all tasks on one processor and balancing the computing costs leads to distribute the tasks on all available processors (if $|T|>|P|$ ).

The cost functions given below correspond respectively to the two previous criteria and try to minimize the most loaded processor (in terms of communication, computation or both):

Minimum communications:

$$
z=\max _{p \in P} \sum_{\mid \text {|alloc }(t)=p} \sum_{t^{\prime} \mid \text { alloc }\left(r^{\prime}\right) \neq p} \operatorname{comm}\left(t, t^{\prime}\right) .
$$

Load imbalance:

$$
z=\max _{p \in P} \sum_{i \mid \operatorname{alloc}(1)=p} \operatorname{calc}(t)
$$

Trade-off:

$$
\begin{aligned}
z= & \max _{p \in P} \sum_{\text {t|alloc }(t)=p}(\operatorname{calc}(t) \\
& \left.+\sum_{t^{\prime} \mid \operatorname{alloc}\left(t^{\prime}\right) \neq p} \operatorname{comm}\left(t, t^{\prime}\right)\right) .
\end{aligned}
$$

This criterion that we have chosen to consider is a trade-off between both previous criteria.

In the cost functions previously described, no overlap between communications and computations was considered. If all communications can occur at the same time as computation (i.e., with the help of specialized processors), the cost function could be adapted by trying to find the maximum between the processor which communicates the more and the processor which computes the more, giving a second cost function. In fact, the cost function should be adapted following the characteristics of the target machine (for instance, distance between processors can be taken into account by analyzing routing tables, etc.).

\section{Modeling parallel algorithms in ANDES}

Parallel algorithms are modeled in ANDES as precedence valued DAGs. The graph vertices model computations and the arcs model the precedence and possibly a communication between computations. A numerical value is associated to the vertices in order to quantify a processing load (e.g., number of instructions to be executed). Also, a numerical value is associated to the arcs, in order to quantify a communication load (e.g., number of integers exchanged). These values are closely related to the application costs. They can be converted to a normalized cost, for example to costs expressed in time units, if a parallel machine model is associated. A more detailed description of ANDES can be found in [8].

The DAG used in ANDES is not given "as is" (for example, as a communication matrix or as a formatted file). Indeed, it is extracted from a more abstract textual description which models a family of parallel algorithms. This abstract description, named $D G-A N D E S$, is a $C$ program using a specific library, which allows the representation of computation nodes and of precedence between these computation nodes. A computation node is more than a single task: it is composed of an input, of an output and of a computation description. These annotations allow the modeling of more complex relationships among the tasks. The concept of "computation" is variable. It may be a single arithmetic operation or a complex algorithm.

The basic type in a DG-ANDES is Node. This type is needed when declaring the computation nodes of a parallel algorithm model and it is used as a classical $\mathrm{C}$ data type. Two basic functions are used to build the graph: comp_node and prec. comp_node identifies the input, the computation and the output descriptions. For example,

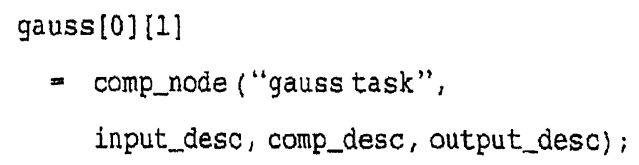


creates the computation node gauss [0] [1] (of Node type), whose input, computation and output descriptions are input_desc, comp_desc and output_desc. These descriptions are classical $\mathrm{C}$ procedures. The string "gauss task" is a comment.

prec is the procedure which defines a precedence between two computation nodes. For example,

$$
\begin{aligned}
& \text { prec("Precedence", gauss }[0][1] \text {, } \\
& 0 \text {, gauss }[1][1], 3 \text { ); }
\end{aligned}
$$

defines a precedence between gauss [0] [1] (output port 0) and gauss [1] [1] (input port 3). Input and output ports numbers identify specific input and output precedences of a computation node and they are important if a reference is needed. For example, it may be necessary to model constructions like the Occam2 alternative where some action is taken depending on which channel a message arrived. In this case, the identification of the port is necessary in order to associate the port with the corresponding action. The string "Precedence" is a comment.

Finally, there are three methods used for input, computation and output descriptions: type_input, type_oper and type_output. For example,

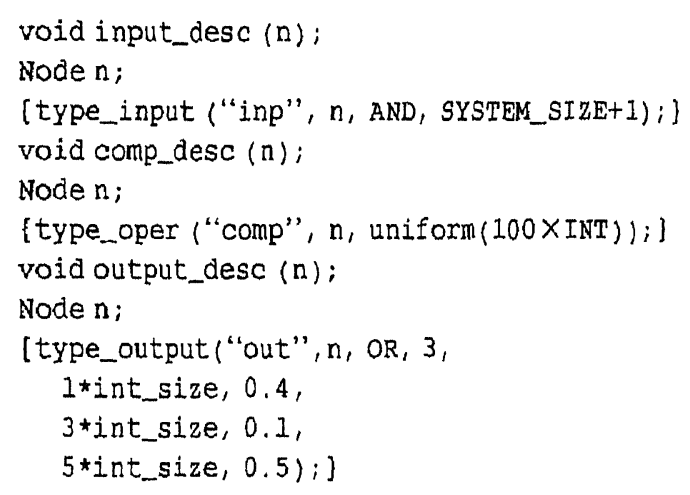

details the input, computation and output descriptions for the previous gauss [0] [1] computation node (see above paragraphs, in the example of comp_node). The strings "inp", "comp" and "out" are comments. The parameter $\mathrm{n}$ is a formal parameter used by the library. This variable is a pointer to the current computation node being described. It is important because the same input/output/computation description can be used by different computation nodes. In the above example of type_input, an AND input with SYSTEM_SIZE+ 1 inputs (SYSTEM_SIZE being a constant of the application description) is described. This means that all the inputs of gauss [0] [1] should arrive before executing the computation associated with the computation node gauss [0] [1]. In comp_desc, a computation of average 100 integer instructions, distributed uniformly, is represented. Finally, in output_desc, an output of type $O R$ is described. This kind of output means that only one output will be chosen: an output of 1 integer with probability 0.4 , an output of 3 integers with probability 0.1 , or an output of 5 integers with probability 0.5 .

Originally, three types of inputs/outputs can be described: (1) Boolean descriptions (like AND, OR). An AND input models a join of control threads, and an $O R$ input models an Occam2 alternative [11]. An AND output models a fork of control threads, and an OR output models the $C$ case instruction; (2) global operations, like data broadcast. A data broadcast can be considered as an AND output, but the data are the same for each output; and (3) grouping input/output, that is, two computation nodes linked by a grouping should be executed by the same processor. This kind of description is important to model, for example, sequential pieces of code that share the same address space and that should be mapped to the same processor. Another application of grouping inputs/outputs is the possibility of clustering several tasks of the graph in order to study the impact of this grouping on the final performance of the application. In other words, grouping task is useful to perform a granularity analysis of a parallel program.

The quantitative costs can also be described as variable quantities: (1) as a constant distribution, in order to model well-known workload, as for exam- 
ple, message sizes; (2) random variables, in order to model, for example, branching probabilities; and (3) dependent variables, that is, costs that depend on other costs of the graph or on the inputs of the algorithm.

Very compact descriptions can be created using the native iterative constructions of the $C$ language. Also, the $C$ random function can be employed to create random variables. Standard structures of algorithms (e.g., trees, grids, diamonds) can be "canned" in procedures which also allow the representation of recursive and hierarchical constructions. In a $D G$ ANDES, a loop is unrolled in order to fit the directed graph representation. The iterative instruction of the host language are suitable for the representation of loops. For example,

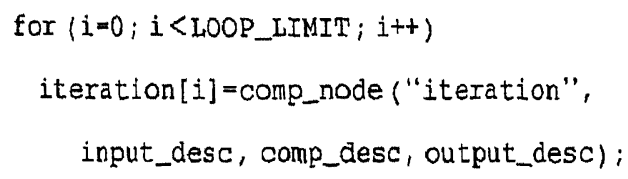

describes a loop in the model. When compiling and executing the program describing the model, the for instruction is executed and consequently the modeled loop is unrolled. The constant LOOP_LIMIT can be substituted by a random function or a value related to another attribute of the DG-ANDES.

The DG-ANDES is then a description of a family of parallel algorithms. Indeed, in a DG-ANDES, uncertainties can be modeled (e.g., average costs, branching probabilities, OR outputs). A DG-ANDES can be compiled and executed (it is a $C$ program). For the work presented here, this execution produces a DAG with all the vertices having AND inputs and outputs and all the (computation and communication) costs being constants. This means that all uncertainty is removed. The DAG is used by the mapping strategy and by the synthetic execution manager in order to produce a synthetic execution from which the required performance indices are computed.

\section{ANDES and the synthetic execution manager}

From the DAG until the synthetic execution, $A N$ $D E S$ performs four main steps: (1) cost conversion, that is, given a parallel machine model, the graph costs (e.g. number of instructions to be executed, the number of exchanged integers) are reduced to the same unit (e.g., microseconds); (2) the DAG clustering; (3) the choice of which processor will execute each cluster (execution of the mapping strategies); and (4) file treatment in order to be read by the synthetic execution manager. These four steps plus the compilation of the DG-ANDES are executed sequentially on a Sparc Sun/4 workstation. The synthetic execution manager executes on the Meganode, a DMPM with 128 Transputers and a statically reconfigurable interconnection network. This target machine is used due to its availability in the context of this research, but another DMPM could be used instead, with little changes.

\section{I. DAG pre-processing}

The application quantitative model given to a mapping algorithm is a valued undirected graph, where the vertices model processes and the edges model communication between processes (see Section 3). The DAG is a richer application model, due to the precedence. However, it does not seem sensible to give a DAG for a mapping strategy. Therefore, a clustering algorithm is used to group the computation nodes of the DAG into clusters. In ANDES, clustering is done using the PYRROS DSC (Dominant Sequence Clustering) Algorithm [19]. The DSC algorithm "performs a sequence of clustering refinement steps and at each refinement step, it tries to zero an edge to reduce the parallel time" [19]. It has $O((v+e) \log v)$ time complexity and $O(v+e)$ space complexity ( $v$ is the number of DAG tasks and $e$ is the number of arcs). Clustering is done considering an unbounded number of processors of a completely 
connected architecture. The mapping strategies presented in Section 3 are then used to choose which processor executes each cluster. Clustering in this study is only used to adapt the models described by a directed graph in an understandable representation for the mapping algorithms (undirected graphs). Currently, no detailed study is conducted in order to compare different clustering strategies and the impact of these strategies on the application performance. Finally, the DAG and the mapping information are stored in a file that is read by the synthetic execution manager running on the Meganode. It is important to remark that during this DAG preprocessing, there is an available Meganode computation and communication model which allows estimation of the computation and communication costs (expressed in time units) used by the clustering and mapping algorithms. These models are linear functions that associate a number of operations to time in computation models and message size and topology to time in communication models. During this phase, it is also possible to estimate the total computation demand of the DAG tasks (their costs and the machine computation model are known - see tap below).

\subsection{Synthetic execution}

The synthetic execution performed in order to obtain the desired performance indices is executed on the Meganode (Fig. 1), a DMPM that contains 128 Transputers (T800/20 MHz and $1 \mathrm{Mb}$ of memory - communication links adjusted to $10 \mathrm{Mbits} / \mathrm{s}$ ), interconnected by a hierarchical and reconfigurable network. The 128 Transputers are grouped in 4 tandems of 32 processors each. There is an internal switch connecting these 32 processors. Another switch interconnects the 4 tandems. This second level switch also interconnects the tandems to the host machine, a Sparc Sun/4 with a 4 Transputer board (each Transputer is a T800/20 MHz and $4 \mathrm{Mb}$ of memory), 3 of them used for program develop-

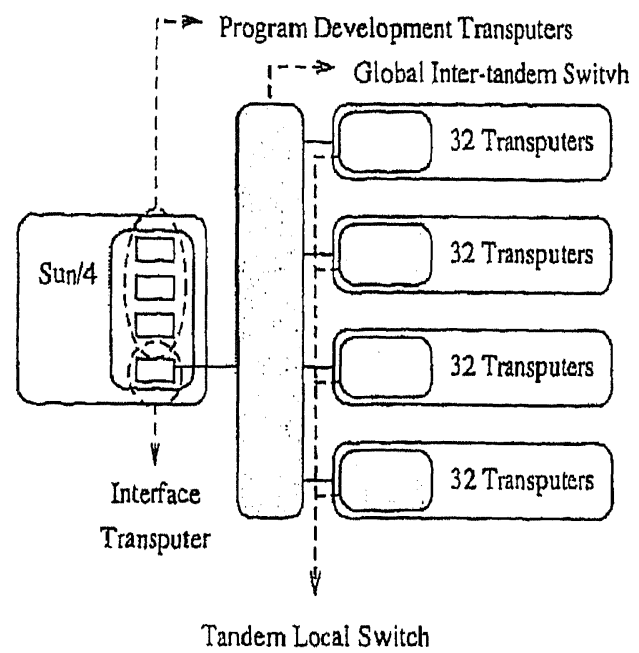

Fig. 1. The Meganode.

ment and only one connected to the Transputer network. The available Meganode is a single-user machine: there is no operating system that allows more than one application to execute on it. The available programming languages are Occam2 and $\mathrm{C}$ (mainly the InmosC toolset IMS-D4214). Routing is done by software using VCR (Virtual Channel Router), Version 2.0k [5].

The synthetic execution manager is the kernel that controls the synthetic execution on the parallel computer. It is a SPMD parallel program written in Inmos $\mathrm{C}$ using routing facilities provided by VCR. There are two types of processes (Fig. 2): one executing on the root interface Transputer inside the host workstation (the root task) and one execuled on each Transputer of the network (the manager tasks). These tasks (all the manager tasks and the root) are virtually completely connected, that is, there is a virtual communication channel between any pair of processes.

The root process reads the application quantitative DAG plus the mapping information and sends, to each manager process on the network, the information of the nodes (tasks) of the DAG mapped on the receiver manager process. In this way, each 


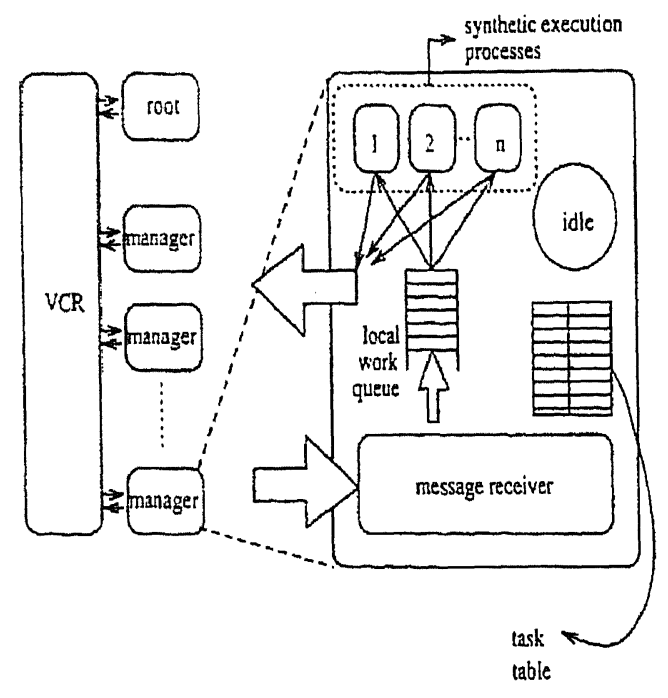

Fig. 2. Process stucture of the synthetic execution manager.

manager has a partial knowledge of the complete DAG. The root keeps the processor identification of the DAG tasks which do not have predecessors. After each network processor has received from the root processor all the information of the tasks mapped on it, the root process starts the synthetic execution and measures time. This root process sends a starting signal to all Transputers containing DAG tasks with no predecessors. After this communication, the root waits for a terminal signal from all manager processes, stops measuring time and receives from all manager processes the load information.

The manager process is composed of two distinct parts. The first one receives, from the root process, the DAG information (computation and communication costs, number of predecessor tasks, number of successor tasks) and puts them into a table (the T-Table). The second part consists of effectively managing the synthetic execution. It is only started if there are tasks placed on the associated processor. Three types of (sub)processes are created just before managing synthetic execution (Fig. 2):

1. a message receiver process: this process receives all the messages for the tasks residing on the processor. When a message arrives, this process verifies the identification of the receiver DAG task. The local DAG T-table is then consulted in order to check whether all the incoming messages for the receiver tasks have arrived. If not, the incoming message counter is decremented, and the process waits for a new message. If yes, a synthetic task execution is signaled (through a local work queue) to the synthetic execution process;

2. a set of synthetic execution processes: when a DAG task is to be executed, the message receiver process informs one of the synthetic execution processes that a synthetic DAG task can be executed. This last process loops for a specific amount of time defined by the application quantitative DAG and the output communications are done. Opposite to the reception of messages, there is not a process that manages the emission of messages: the synthetic execution process itself sends the appropriate messages to the successor DAG tasks. One important remark is that the number of synthetic execution processes is specified by the user of the kernel. This parameter is known as the "multiprogramming degree", and the higher is this degree, the more exploited is the Transputer time-sharing capacity;

3. an idle process: this process runs only when no other process is running (including the VCR internal processes). The idle process only increments a counter. The final value of this counter is used to estimate the processor idle time.

After the execution of the kernel, some data are obtained concerning the execution of the synthetic application. The time of the synthetic execution $\left(T_{e x}\right)$ is measured. The time spent by the iterations of the idle process in processor $p\left(t\right.$ idle $\left._{p}\right)$ can be obtained through the linear model of number of iterations versus execution time, specific for a given processor. In this way, $t$ work $_{p}=T_{\mathrm{ex}}-t$ idle $_{p}$ gives the time a processor $p$ worked. $t$ work $_{p}$ can still be 
decomposed in two parts: (1) the time a processor spends executing the computations modeled by the input DAG $\left(\operatorname{tap}_{p}\right)$; and (2) the time a processor spends communicating, managing the synthetic execution and doing housekeeping (the overhead time $\left.t_{0} h_{p}\right) t_{a p}$ is computed before the synthetic execution. toh $p$ is then computed doing $T_{e x}-\operatorname{tap}_{p}-$ tidle $_{p}$, for each processor $p$. For a given application, the estimated overhead on processor $p$ in microseconds due to the tool (not taking into account VCR and communication overheads) is $204 \times \mathrm{NT}+215$ $\times \mathrm{MD}+140 \times \mathrm{MSGA}+40 \times \mathrm{MSGD}+80$, where NT is the number of tasks placed on $p, M D$ is the "multiprogramming degree" on $p, M S G A$ is the number of arriving messages in $p$, and finally, MSGD is the number of messages leaving $p$.

\section{Evaluation of the mapping strategies}

ANDES is then used in the evaluation of task mapping strategies. In this experimental approach, the set of experiments described below has been performed. The starting point is a benchmark composed of 17 models of parallel algorithms ( $D G$ ANDES). This benchmark is derived from different sources (the literature and real benchmarks). We hope that it is representative for scientific computing. The models are of (1) the Bellman-Ford iterative algorithm for computing the path length in a graph; (2)-(5) 4 systolic diamond-shaped computations; (6) a divide-and-conquer; (7) one-dimensional FFT; (8) Gaussian elimination (9) a generic iteration; (10) master-slave; (11) master-slave followed by Gaussian elimination (this model tries to model an irregular application); (12)-(13) two partial differential equation iterative algorithms; (14) a tree computation; (15) a quantum dynamics algorithm; (16) the recursive Strassen algorithm for matrix multiplication, and (17) the Warshall algorithm for finding the transitive closure of an adjacency matrix. For each program of this benchmark, some parameters are considered important for performance evaluation: the number of groups generated by PYRROS, the total computation cost, the total communication cost (and the ratio computation/communication), the standard deviation of the costs among the tasks, the number of inputs / outputs of a group, its granularity (i.e., the mean time interval between two extermal communications of a group) and its virtual parallelism (the width of the DG-ANDES). For example, executing the program corresponding to the ANDES model of the Strassen benchmark, the following values for the main parameters are obtained: 1140 groups, a total computation cost of $8,623,185$ microseconds, a total communication cost of $11,564,613$ microseconds and a virtual parallelism of 92.69 .

There are four types of cost functions:

- $A D D$ is an additive cost function (non-overlap of computation and communication);

- MAX is a maximum cost function (overlap computation-communication);

- ROUT considers non-overlap of computation and communication and a store-and-forward routing;

- TOR considers non-overlap of computation and communication and a packet switching routing.

Considering the above 4 cost functions used to evaluate the quality of the placement, there are 4 greedy strategies (modulo, LPTF, LPTF with a quantitative criterion, and LPTF with a structural criterion). There are also 2 iterative algorithms: a simulated annealing and a tabu search, both starting from a solution defined by a greedy algorithm (LPTF with a quantitative criterion). These algorithms are available in our research environment. Each model of the benchmark, clustered automatically or manually, is given to each mapping strategy and the corresponding synthetic charge is executed 100 times in order to obtain an average execution time. For each model, the communication/computation ratio is modified three times. The total number of different workloads is 102 . 
The target architecture is the Meganode multicomputer itself, configured as a $4 \times 4$ torus. Topologies with more processors are considered (larger tori) in order to evaluate the scalability of the obtained measures. Also, the multiprogramming degree can be changed (if more than one task of the DG-ANDES is
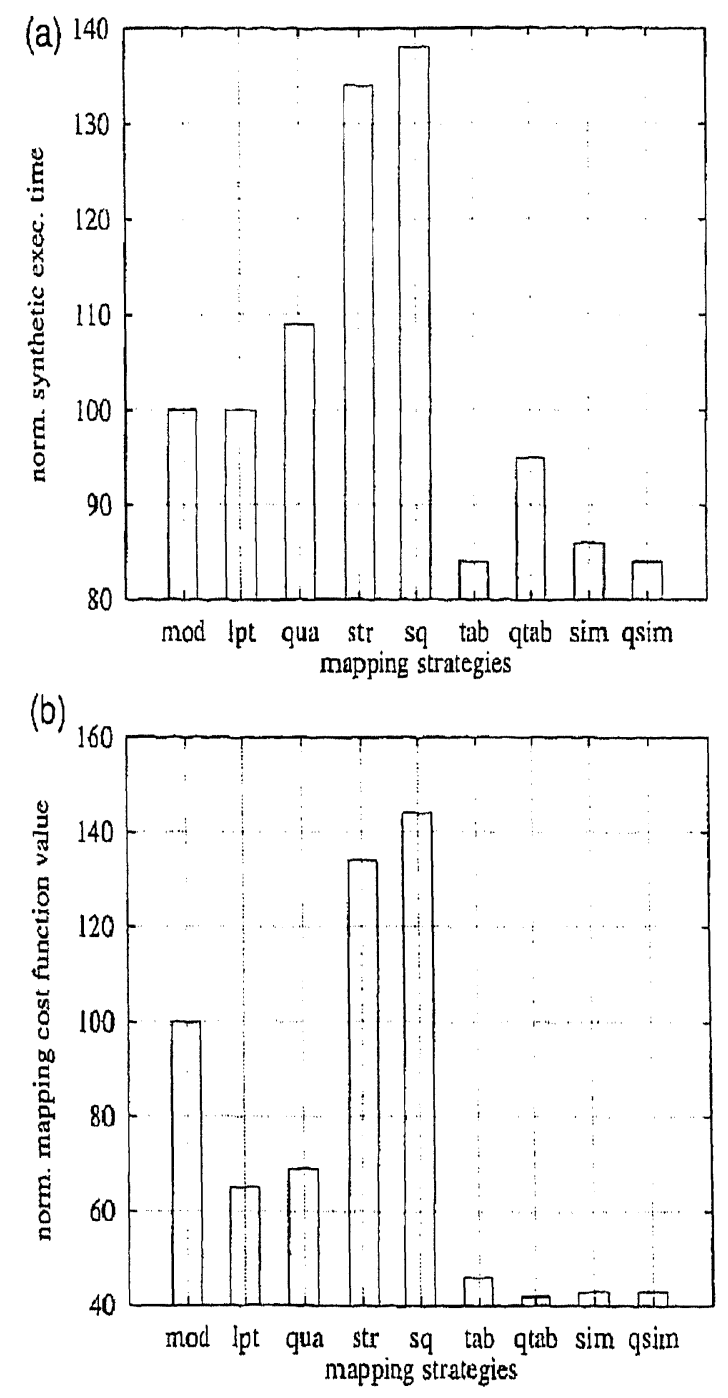

Fig. 3. Synthetic execution time and cost function values per mapping strategy for Strassen benchmark $(\bmod =$ modulo, $l p t=$ standard lpt, qua $=$ quantitative lpt, str $=$ structural lpt, $s q=$ structural and quantitative lpt, tab =tabu, qtab $=$ tabu from a quantitative solution, sim = simulated annealing, qsim $=$ simulated annealing from a quantitative solution). able to execute, they are executed in a time-sliced fashion).

Finally, the following indices can be obtained when using ANDES: the value of the cost function of the final solution given by the mapping strategies, the execution time of the mapping strategy, the execution time of the synthetic program, the fraction of the execution time corresponding to the execution of the application tasks, the fraction corresponding to the overhead (due to the communication and to the tool itself) and the fraction corresponding to the idle time. The performance indices that can be analyzed when using these strategies are the distribution of the groups on the processors and the reduction of the communication costs between groups due to the mapping (the communication cost between two groups mapped to the same processor is considered null).

For example, graphics like those presented in Fig. 3 support the comparison between the synthetic execution and the performance given by the mapping strategies (the values are normalized: the reference value is that given by the modulo strategy). Fig. 4 presents an example of a graph representing the load of the machine during the synthetic execution. On a given bar, the lower region represents the useful work, the middle region corresponds to the overhead and the upper region corresponds to the idle time of the processor. The large overhead fraction is caused by an excessive degree of the application communications, considering that the overhead caused by the tool is forced to be inferior to $10 \%$ of the total computation cost of the program model.

\subsection{Results}

A linear regression was performed between the set of cost function values (which estimates the execution time of the synthetic workload) and the set of measured execution times. The cost function TOR which represents well the behavior of the Meganode is the best cost function. The linear model given by 


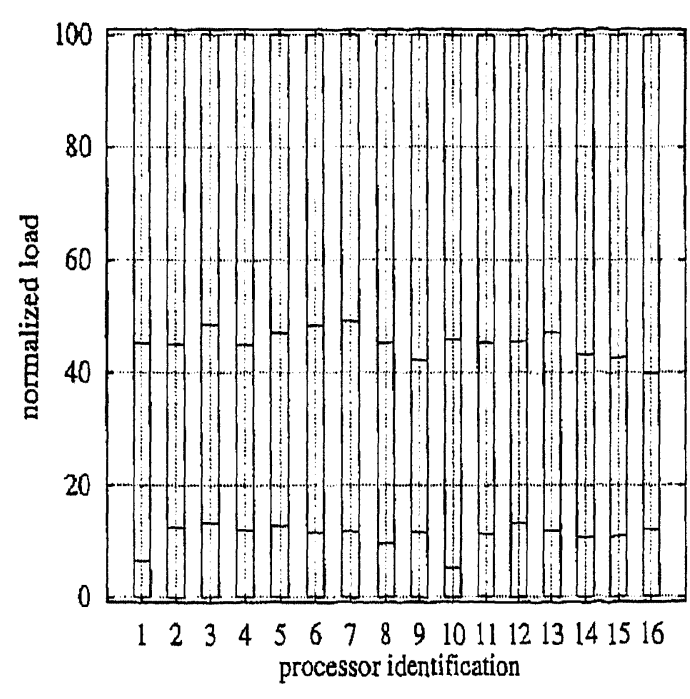

Fig. 4. The load profile of the Strassen benchmark for qtabu mapping strategy.

the regression is the closest if compared with the execution time (i.e, cost function value close to the measured execution time). The cost function $\mathrm{ADD}$ (non-overlap of communication and computation) is also good. Table 1 gives the slope of the obtained linear model and the quality of the regression (a good regression has quality close to 1 ).

Considering a given program model (among 17), a given communication/computation ratio (there are 3 for each model) and a given clustering (there are 2 types of clustering), 6 mapping strategies were applied. In $76 \%$ of the cases, the worst mapping algorithms (the algorithm that gives the worst execution time) are modulo and LPTF. In $12 \%$ of the cases, modulo and LPTF are not the worst strategies but the

Table 1

\begin{tabular}{lccccl}
\hline & ADD & MAX & TOR & ROUT & Clustering \\
\hline Slope & 1,213 & 1,620 & 1,185 & 1,636 & Pyrros \\
Quality & 0,923 & 0,894 & 0,907 & 0,947 & Pyrros \\
Slope & 1,370 & 2,147 & 1,302 & 1,664 & Manual \\
Quality & 0,734 & 0,796 & 0,794 & 0,771 & Manual \\
\hline
\end{tabular}

difference between the best strategy and the worst is not superior to $10 \%$. When this difference is greater than $10 \%$, the worst strategy is iterative (it may go to a local minimum that is not a global minimum). The conclusion is that all the strategies that do not consider the communication as mapping criterion (modulo and LPTF) are not good in practice. In $81 \%$ of the cases, the best strategy is one that considers the existence of the communications. We verify also that the best speed-ups (the ratio between the total sum of the computation costs of the graph and the measured execution time) are obtained for the models having the lowest communication/computation ratio. A very interesting conclusion is that the iterative algorithms do not improve reasonably the mapping given by a greedy algorithm. It is verified that for the given benchmark the improvements obtained with the simulated annealing and the tabu are inferior to $10 \%$. A possible reason of this behavior is the regularity and symmetry of the 17 models. A modification of a already done mapping (e.g., by pair exchanging) on these program models do not represent a considerable gain in terms of execution time. Taking into account that a benchmark is a representative set of models of real programs, a greedy mapping algorithm is enough to map tasks on processors.

\section{Conclusion and perspectives}

ANDES is a performance evaluation tool based on synthetic programs and was developed initially for support of the evaluation of different mapping strategies. The tool is being used intensively in order to acquire knowledge of the strategies behavior. The goal is to obtain some rules of thumb about the choice of the best mapping strategy given a specific parallel program and a specific parallel architecture. However, ANDES has been designed for wider use. Other implementation and execution strategies can be evaluated like scheduling and load balancing, 
implying a change of the synthetic execution manager.

The choice of the synthetic approach was done in order to take into account the real overheads of the execution of a parallel program on a parallel machine. These overheads (for example, those associated with the communication system of a parallel machine) are sometimes difficult to model when using analytical and simulation models. In this way, ANDES allows performance evaluation at the model level, but with some realistic (or almost realistic) components. This experimental approach is rather new, considering that normally mapping strategies are compared according to different values of the cost function [13].

Future work is planned. ANDES currently runs on a Transputer machine. It will be ported on the IBM SP multicomputer. With the SP version, mapping, scheduling and load balancing strategies will be evaluated. A toolbox of the best strategies will compose the kernel of the parallel programming environment currently being developed inside the APACHE project. This environment is based on Athapascan, a programming language based on Remote Procedure Calls. Later, ANDES will be used inside this programming environment as a tool used for performance prediction. With the version on the SP, $A N$ DES models will be described using $\mathrm{C}++$ (instead of $\mathrm{C}$, as done today). This language seems to be more adequate to model objects like tasks. $\mathrm{C}++$ will also be used to describe machine models.

\section{Acknowledgements}

Meganode is a Telmat trademark. Sun and Sparc Sun $/ 4$ are Sun Microsystems Inc. trademarks. Occam, Occam2 and Transputer are Inmos, Inc. trademarks. The ANDES environment is related to the ALPES (ALgorithms, Parallelism and Evaluation of Systems) research group inside the APACHE project. The ALPES group is supported by the CNRS,
INPG, PRC $C^{3}$, MESR, $\mathrm{CNPq} /$ Brazil and the Rhône-Alpes Region.

\section{References}

[1] Shahid H. Bokhari. On the mapping problem. IEEE Transactions on Computers, C-30(3):207-214, March 1981.

[2] S.W. Bollinger and S.F. Midkiff. Processor and link assignment in multicomputers using sinulated annealing, In 1988 International Conference on Parallel Processing, University Park, The Pensylvania State University Press, 1988.

[3] Rosemary Candlin and Neil Skilling. A modelling system for the investigation of parallel program performance. In: Gian. franco Balbo and Giuseppe Scrazzi, eds., Computer Perfor. munce Evaluation: Modelling Techniques and Tools, pages 397-409, Elsevier, Amstcrdam, 1992.

[4] T.L. Casavant and J.G. Kuhl. A taxonomy of scheduling in general-purpose distributed computing systems. IEEE Trans. actions on Sofnware Engineering, SE-14(2):141-154, Febaary 1988.

[5] Mark Debbage, Mark B. Hill and Denis A. Nicole. The Virtual channel router. Transputer Communicutions, 1(1):318, August 1993.

[6] Fred Glover, Tabu search: A tutorial. Interficess, 20(4);74-94, July 1990.

[7] H. Kasahara and S. Narita. Prictical multiprocessor scheduling algorithms for efficient parallel processing. IEEE Trans. actions on Computers, 1984.

[8] Joāo Paulo Kitajima, Cécile Tron and Brigitte Plateaul. ALPES: a tool for the performance evaluation of paralle programs. In: Jack J. Dongarla and Bemard Tournncheat eds., Environments and Tooks for Parallel Scientific Compuring, pages 213-228, North-Holland, Amsterdan, 1993.

[9] C-L. Lee. Parallel machines scheduling with nonsimultaneous machine available time. Discrete Applied Mathemaniss, 1991.

[10] Ted G. Lewis and Hesham El-Rewini. Introduction to Paral. lel Computing. Prentice-Hall International, Englewood Clifise, 1992.

[11] INMOS Limited. Occam 2 reference mamual. Prentice-Hal Intermational Scries in Compuler Science. Prentice-Hall International, New York, 1988.

[12] Virginia M. Lo, ct al. OREGAMI: softwarc tools for map ping parallel computalions to parallel architeclures. Tcchnical Report TR-PPSE-89.13, OACIS, 19500 H.W. Gibbs Drive, Suite 110, Beaverton, OR, USA, 1989.

[13] Michael G. Norman and Peter Thanisch. Models of machines and computation for mapping in multicomputers. ACM Computing Surveys, 25(3):263-302, September 1993. 
[14] Jean-Louis Pazat. Outils pour la programmation d'un multiprocesseur à mémoires distribuées. Ph.D. Thesis, Université de Bordeaux I, 1989.

[15] P. Pouzet, J. Paris and V. Jorrand. Parallel application design: the simulation approach with HASTE. In: Wolfgang Gentzsch and Uwe Harms, eds., Proceedings of HPCN'94, Lecture Notes in Computer Science - 797, pages 379-393, München, BRD, 1994. Springer-Verlag.

[16] Harold S. Stone and Shahid H. Bokhari. Control of distributed processes. Computer, 11(7):97-106, July 1978.

[17] V.S. Sunderam, G.A. Geist, J. Dongarra and R. Manchek. The PVM concurrent computing system: evolution, experiences, and trends. Parallel Computing, 20(4):531-546, April 1994.

[18] Stephan Waser and Helmar Burkhart. OLGA - A modelling tool for algorithmic skeletons. In: Reinhard Grebe et al., eds., Transputer Applicutions and Systems '93, Volume 1, pages 395-409, IOS Press, Amsterdam, 1993.

[19] Tao Yang and Apostolos Gerasoulis. PYRROS: Static scheduling and code generation for message passing multiprocessors. In Proceedings of the 6ih ACM International Conference on Supercomputing, pages 428-437. ACM, July 1992.

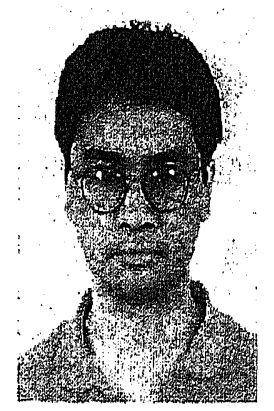

João Paulo Kitajima received a Master degree in Computer Science from the Universidade Federal do Rio Grande do Sul, Brazil, in 1990, and a Doctorate degree in Computer Science from the Institut National Polytechnique de Grenoble, France, in 1994. He worked as an associated researcher at Universidade de Brasilia, Brazil, during 1995 , and, since February 1996, he holds a position of professor in the Universidade Federal de Minas Gerais, Brazil. His research interests are parallel computing and performance evaluation.

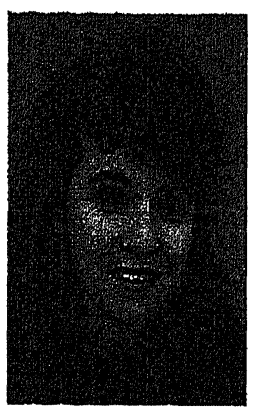

Brigitte Plateau received a Master in Applied Mathematics from the University of Paris 6 in 1976, a Thèse de Troisième Cycle in Computer Science from the University of Paris 11 in 1980, and a These d'Etat in Computer Science from the University of Paris 11 in 1984. She was Chargé de Recherche at CNRS (France) from 1981 to 1985, Assistant Professor at the Computer Science Department of the University of Maryland from 1985 to 1987. She currently holds a position of Professor at the engineering school ENSIMAG in Grenoble (France) and is a member of a research group whose main interest is the study of massively parallel machines. Her research interests include queuing theory and performance evaluation of parallel and distributed computer systems.

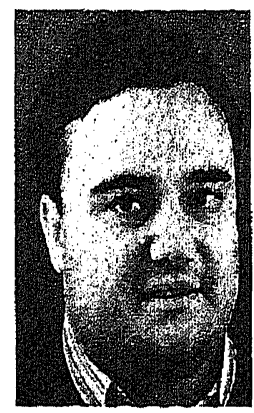

Pascal Bouvry received his M.Sc. Degree in computer science from FUNDP, University of Namur, Belgium, in 1991 and his Ph.D. degree in computer science from INPG, university of Grenoble, France, in 1994 at the LMC-IMAG laboratory. He is currently Senior Researcher at CWI (Center for Mathematics and Computer Science) in Amsterdam, the Netherlands. His research interests include Concurrent/Parallel Programming Environments and Languages, Visual Programming and Operations Research.

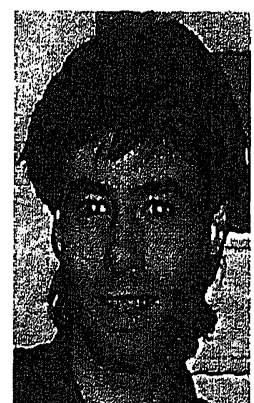

Denis Trystram was bom in Paris in 1958. He has received two Ph.D. from the Institut National Polytechnique dc Grenoble respectively in Applied Math. ematics (1984) and Computer Science (1988). He is now Professor in Computer Science at the Institut National Polytechnique de Grenoble since 1991. Since 1990, he is at the Head of the "Parallel Processing" group at LMCIMAG. He manages an interdisciplinary regional group for parallelizing practical applications (ParAppli), and he is currently Regional Editol Parallel Computing Joumal. His research activities concem all aspects of the study of the impact of parallelism on the conception of efficient algorithms (namcly, Model and Design of parallel algorithms, Scheduling and Mapping, Optimization of Communications, Implementation of real applications on parallel machines). He has published five books, and about 50 articles in international joumals and as many international conferences. 